\title{
Article \\ Evaluating Whether Radiofrequency Irradiation Attenuated UV-B-Induced Skin Pigmentation by Increasing Melanosomal Autophagy and Decreasing Melanin Synthesis
}

\author{
Hyoung Moon Kim ${ }^{1,2,+}$, Seyeon Oh ${ }^{2,+}$, Jin Young Yang ${ }^{2}$, Hye Jin Sun ${ }^{3}$, Miran Jang ${ }^{3}$, Donghwan Kang ${ }^{3}$, \\ Kuk Hui Son ${ }^{4, *}$ and Kyunghee Byun ${ }^{1,2, *}$ \\ 1 Department of Anatomy \& Cell Biology, Gachon University College of Medicine, Incheon 21936, Korea; \\ drmac12@me.com \\ 2 Functional Cellular Networks Laboratory, Lee Gil Ya Cancer and Diabetes Institute, Gachon University of \\ Medicine, Incheon 21999, Korea; seyeon8965@gmail.com (S.O.); roswellgirl111@gmail.com (J.Y.Y.) \\ 3 Jeisys Medical Inc., Seoul 08501, Korea; sunhj@jeisys.com (H.J.S.); lyla@jeisys.com (M.J.); \\ kang@jeisys.com (D.K.) \\ 4 Department of Thoracic and Cardiovascular Surgery, Gachon University Gil Medical Center, \\ Gachon University, Incheon 21565, Korea \\ * Correspondence: dr632@gilhospital.com (K.H.S.); khbyun1@gachon.ac.kr (K.B.); \\ Tel.: +82-32-460-3666 (K.H.S.); +82-32-899-6511 (K.B.) \\ + These authors contributed equally to this study.
}

Citation: Kim, H.M.; Oh, S.; Yang, J.Y.; Sun, H.J.; Jang, M.; Kang, D.; Son, K.H.; Byun, K. Evaluating Whether Radiofrequency Irradiation Attenuated UV-B-Induced Skin Pigmentation by Increasing Melanosomal Autophagy and Decreasing Melanin Synthesis. Int. J. Mol. Sci. 2021, 22, 10724. https:// doi.org/10.3390/ijms221910724

Academic Editor: Alessandra Marconi

Received: 8 September 2021

Accepted: 1 October 2021

Published: 3 October 2021

Publisher's Note: MDPI stays neutral with regard to jurisdictional claims in published maps and institutional affiliations.

Copyright: (c) 2021 by the authors. Licensee MDPI, Basel, Switzerland. This article is an open access article distributed under the terms and conditions of the Creative Commons Attribution (CC BY) license (https:// creativecommons.org/licenses/by/ $4.0 /)$.
Abstract: Autophagy is involved in the degradation of melanosomes and the determination of skin color. TLR4 and tumor necrosis factor (TNF) signaling upregulates NF-kB expression, which is involved in the upregulation of mTOR. The activation of mTOR by UV-B exposure results in decreased autophagy, whereas radiofrequency (RF) irradiation decreases TLR4 and TNF receptor (TNFR) expression. We evaluated whether RF decreased skin pigmentation by restoring autophagy by decreasing the expression of TLR4 or TNFR/NF-KB/mTOR in the UV-B-irradiated animal model. UV-B radiation induced the expressions of TNFR, TLR, and NF- $\mathrm{kB}$ in the skin, which were all decreased by RF irradiation. RF irradiation also decreased phosphorylated mTOR expression and upregulated autophagy initiation factors such as FIP200, ULK1, ULK2, ATG13, and ATG101 in the UV-B-irradiated skin. Beclin 1 expression and the expression ratio of LC3-I to LC3-II were increased by UV-B/RF irradiation. Furthermore, melanin-containing autophagosomes increased with RF irradiation. Fontana-Masson staining showed that the amount of melanin deposition in the skin was decreased by RF irradiation. This study showed that RF irradiation decreased skin pigmentation by restoring melanosomal autophagy, and that the possible signal pathways which modulate autophagy could be TLR4, TNFR, NF-kB, and mTOR.

Keywords: melanosomal autophagy; autophagosome; radiofrequency microneedling; ultraviolet B; skin pigmentation

\section{Introduction}

Melanin is a nitrogen-containing pigment made from the melanin precursor L-tyrosine and is deposited in melanosomes, which are subcellular lysosome-like organelles [1]. Both keratinocytes and melanocytes are involved in melanogenesis in the skin: melanocytes produce the melanin and deliver it to the keratinocytes for skin protection [2].

Human skin color is determined by the balance between melanin synthesis and degradation [3]. Microenvironment alterations caused by ultraviolet (UV) radiation, free radicals, and inflammation change this balance, and such changes eventually result in either skin depigmentation or hyperpigmentation $[4,5]$.

Melanogenesis is initiated by UV via the melanocortin-1 receptor [6]. By binding the $\alpha$-melanocyte-stimulating hormone $(\alpha-\mathrm{MSH})$ to MC1R, the melanocyte-inducing transcrip- 
tion factor (MITF) is activated and increases tyrosinase, which is a rate-limiting enzyme of melanogenesis [6].

Autophagy is a vital cellular catabolic system for maintaining tissue homeostasis that eliminates aggregated or misfolded proteins and dysfunctional organelles [7]. Autophagy is also involved in melanosome degradation. Melanosomes are degraded by autophagy after being transferred from melanocytes to keratinocytes [8]. Moreover, melanosomes accumulated in melanocytes due to disrupted transport are also degraded by autophagy [9].

Solar UV upregulates the mammalian target of the rapamycin complex (mTORC), which inhibits unc-51-like autophagy-inhibiting kinase (ULK) 1 and decreases the complex formation of ULK1 with autophagy-related protein (ATG) 13 and the $200 \mathrm{kDa}$ familyinteracting protein (FIP200), thereby decreasing autophagy [10].

The activation of nuclear factor kappa-light-chain-enhancer of activated B cells (NF$\mathrm{kB})$ as an upstream target of mTORC1 inhibits autophagy [11-13]. Furthermore, the canonical NF- $\mathrm{kB}$ pathway is activated by pro-inflammatory pathways like the tumor necrosis factor (TNF) receptors, Toll-like receptors (TLR), and antigen receptors [14,15]. TNF has been shown to induce NF- $\mathrm{KB}$ activation, thereby decreasing autophagy in various cancer cells such as Ewing's sarcoma, breast, and leukemia cancer cell lines. Conversely, the downregulation of NF- $\mathrm{KB}$ reactivates the autophagy process [11,16]. TLR4, an upstream target of NF- $\kappa \mathrm{B}$, is also known to dysregulate mTORC-dependent autophagy [17].

UV-B increases the production of various inflammatory molecules such as interleukin (IL)- $1 \alpha$, IL-1 $\beta$, IL-6, IL-8, and TNF- $\alpha$ in keratinocytes [18-20]. UV-B-irradiated keratinocytes also secrete high-mobility group box 1 (HMGB1), which is a ligand of TLR4 [21]. These proteins are associated with post-inflammatory hyperpigmentation, which results from the overproduction or irregular dispersion of melanin [22].

Radiofrequency (RF) irradiation has been shown to decrease IL-6, IL-8, and HMGB1 expression in UV-B-irradiated keratinocytes [21], as well as the expressions of NF- $\mathrm{kB}$, TNF- $\alpha$, and TLR4 in the UV-B-irradiated animal model, thereby decreasing keratinocyte proliferation and pigment accumulation [21]. RF could decrease skin pigmentation by modulating skin inflammation through the downregulation of various pro-inflammatory signals like NF- $\mathrm{kB}, \mathrm{TNF}-\alpha$, and TLR4.

It is well known that UV-B irradiation increases various inflammatory cytokines and increases NF- $\mathrm{kB}$, which eventually decreases melanosomal autophagy and increases skin pigmentation. Even though it is known that RF could decrease skin pigmentation, research concerning the mechanism of how skin pigmentation could be decreased by RF has mainly been focused on modulating inflammation. We thought it could be possible that RF decreases skin pigmentation by decreasing skin inflammation, which eventually leads to an increase in melanosomal autophagy. We hypothesized that RF downregulates TNF- $\alpha$ and TLR4, thereby downregulating NF- $\mathrm{kB}$, and decreasing mTOR expression to restore melanosomal autophagy. In this study, we investigated the effects of RF irradiation on autophagy and skin pigmentation in the UV-B radiation animal model.

\section{Results}

\subsection{RF Decreased the Expressions of TNFR, TLR4, and NF- $\kappa B$}

First, we evaluated whether RF irradiation decreased the expressions of TNFR, TLR4, and NF- $\mathrm{KB}$ in the UV-B-irradiated human primary epidermal keratinocytes (HEKn cells). The expressions of TNFR, TLR4, and NF- $\mathrm{KB}$ were significantly increased by UV-B radiation in HEKn cells, whereas RF irradiation significantly decreased them at $24 \mathrm{~h}$ after RF irradiation (Figure 1A-D). 
A
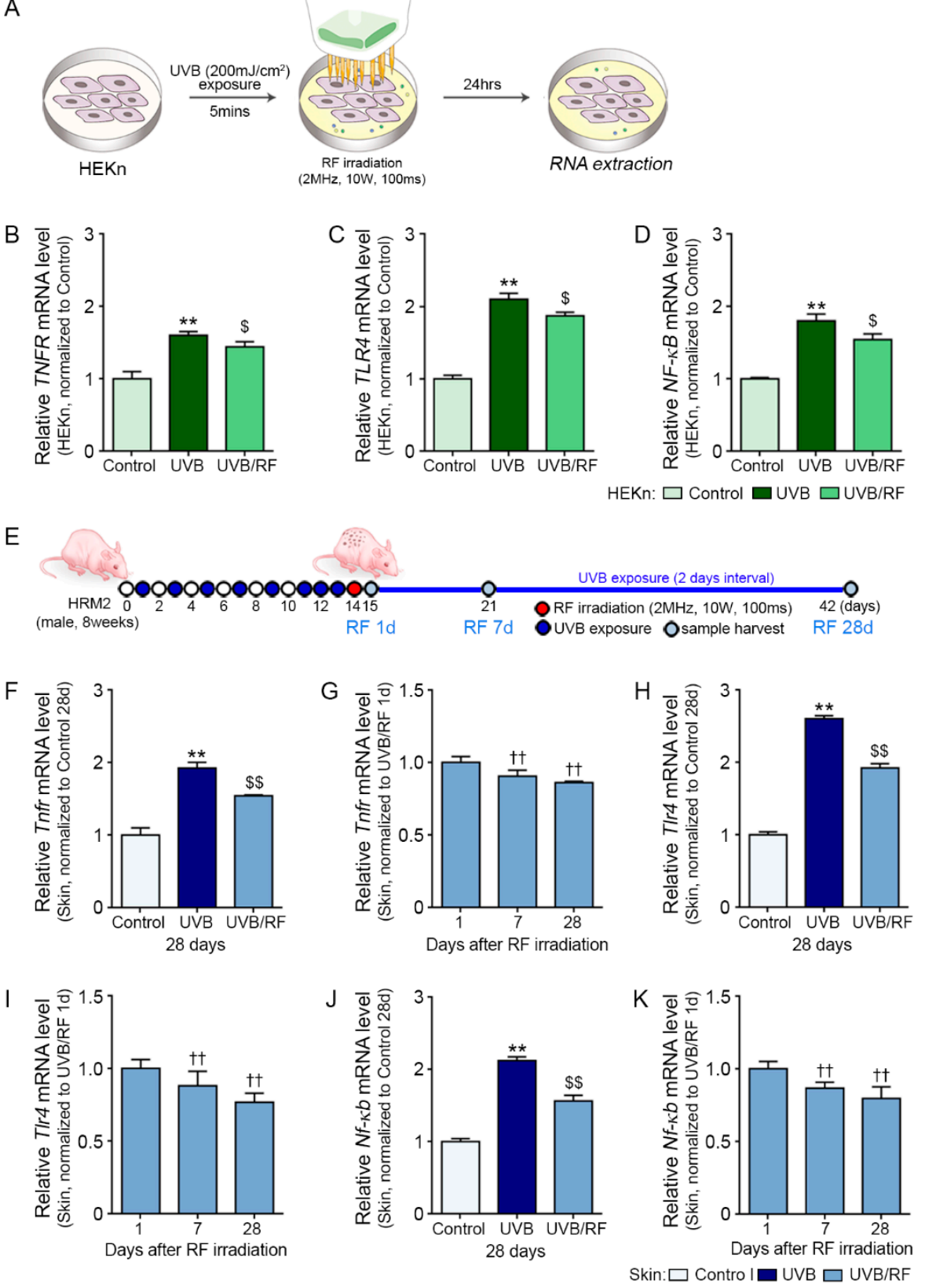

Figure 1. Downregulation of the expressions of TNFR, TLR4 and NF- $\kappa B$ by RF irradiation. (A) HEKn were irradiated either with UV-B $\left(200 \mathrm{~mJ} / \mathrm{cm}^{2}\right)$ or with UV-B/RF (2 MHz, $10 \mathrm{~W}$ for $\left.100 \mathrm{~ms}\right)$ in vitro, or otherwise not irradiated (control). The mRNA expression levels of (B) TNFR, (C) TLR4, and NF- $\mathrm{B}$ (D) were determined in HEKn. The mRNA levels in HEKn were measured by qRT-PCR, normalized versus $\mathrm{ACTB}$, and expressed relative to levels in the control group. (E) Mouse skins were irradiated either with UV-B (200 mJ/ $\left.\mathrm{cm}^{2}\right)$ or with UV-B/RF (2 MHz, $10 \mathrm{~W}$ for $100 \mathrm{~ms}$ ) in vivo, or otherwise not irradiated (control 28 days). The mRNA expression levels of (F,G) TNFR, (H,I) TLR4, and (J,K) NF- $k b$ were determined in the skin tissue. The mRNA levels in the mouse skins were validated by qRT-PCR, normalized versus ACTB, and expressed relative to levels in the control 28 days group or 1 day after RF irradiation. Data are presented as mean $\pm \mathrm{SD}$. ${ }^{* *}, p<0.01$, vs. control (HEKn) or control 28 days (Skin); $\$, p<0.05 ; \$ \$, p<0.01$, vs. UV-B (HEKn) or UV-B 28 days (Skin); $†$, $p<0.01$, vs. 1 day after RF irradiation (Mann-Whitney U test). HEKn, human epidermal primary keratinocytes; NF- $\kappa \mathrm{B}$, nuclear factor kappa-light-chain-enhancer of activated B cells; RF, radiofrequency; TNFR, tumor necrosis factor receptor; TLR4, Toll-like receptor 4; UV-B, ultraviolet-B; UV-B/RF, UV-B plus RF. 
Statistical differences of all factors among the control, UV-B, and UV-B/RF groups were compared at the end of the experiment ( 28 days after RF irradiation). The changes of all factors by time points after RF irradiation were evaluated at 1, 7, and 28 days after RF irradiation, since we wanted to evaluate how long the RF effect continued. The statistical difference among time points was also compared (Figure 1E).

TNFR expression in UV-B-irradiated skin was significantly higher than those in the control and UV-B/RF groups. In the UV-B/RF group, TNFR expression was highest at 1 day after $R F$ but decreased with time (Figure $1 \mathrm{~F}, \mathrm{G}$ ).

The expressions of TLR4 and NF- $\mathrm{kb}$ were significantly increased by UV-B irradiation and were significantly higher than those in the control and UV-B/RF groups. In the UV$\mathrm{B} / \mathrm{RF}$ group, the expressions of TLR4 and NF- $\mathrm{kb}$ were highest at 1 day after RF irradiation but decreased with time (Figure $1 \mathrm{H}-\mathrm{K}$ ).

2.2. RF Decreased mTOR Expression and Induced the Expression of Autophagy Initiation Factors in UV-B-Irradiated Skin

The expression ratio of pmTOR to mTOR was significantly higher in the skins of the UV-B group than those in the control and UV-B/RF groups (Figure 2A,B). In the UV-B/RF group, the ratio of pmTOR to mTOR was highest at 1 day after RF but also decreased with time (Figure 2A-C).

The expressions of autophagy initiation factors, such as FIP200, ULK1, ULK2, ATG13, and ATG101, were significantly lower in the UV-B group than in the control and UV-B/RF groups. In the UV-B/RF group, the expressions did not differ significantly among the time points after RF (Figure 2D-M).

\subsection{RF Increased Melanosomal Autophagy and Degradation}

Beclin 1 expression was significantly lower in the UV-B 28 days group than those in the control 28 days and UV-B/RF 28 days groups. In the UV-B/RF group, the expression of Beclin 1 was highest at 1 day after RF (Figure $3 \mathrm{~A}-\mathrm{C}$ ).

The expression ratio of precursors of microtubule-associated protein light chain 3 (LC3)-like proteins to LC3-II in the UV-B group was significantly lower than that in the control 28 days group and the UV-B/RF 28 days group. In the UV-B/RF group, the expression ratio of LC3-I to LC3-II was highest at 1 day after RF (Figure 3A,D,E).

Melanosomal degradation by autophagy was evaluated by counting the number of melanin-containing autophagosomes in the transmission electron microscopy (TEM).

Melanin-containing autophagosomes were not observed in the control and UV-Birradiated groups. Such autophagosomes observed from 1 day after RF irradiation disappeared at 28 days after RF irradiation. The number of melanin-containing autophagosomes was highest at 1 day after RF irradiation (Figure 3F,G).

\subsection{RF Decreased Skin Pigmentation}

The areas of black spots in the skin of the UV-B group were significantly greater than those in the control and UV-B/RF groups 28 days after RF irradiation. In the UV-B/RF group, the areas of black spots were significantly largest at 1 day after RF (Figure $4 \mathrm{~A}$; upper panel, Figure 4C).

Fontana-Masson staining showed that melanin deposition in the UV-B group was significantly higher than those in the control and UV-B/RF groups. In the UV-B/RF group, melanin deposition was significantly highest at 1 day after RF (Figure 4A; lower panel, Figure 4D,E).

We also evaluated changes of melanin synthesis by RF with $\alpha$-MSH-treated human epidermal melanocytes (HEMn) (F). The expression of MC1R and MITF were significantly increased by treating $\alpha-\mathrm{MSH}$, however those were significantly decreased by RF (Figure $4 \mathrm{G}, \mathrm{H}$ ). Melanin content that was evaluated with a melanin assay was significantly increased by treating $\alpha-\mathrm{MSH}$, however it was decreased by RF (Figure 4I). Images from TEM also showed that $\alpha$-MSH increased melanin in the HEMn and melanin was decreased by RF (Figure 4J). 
A
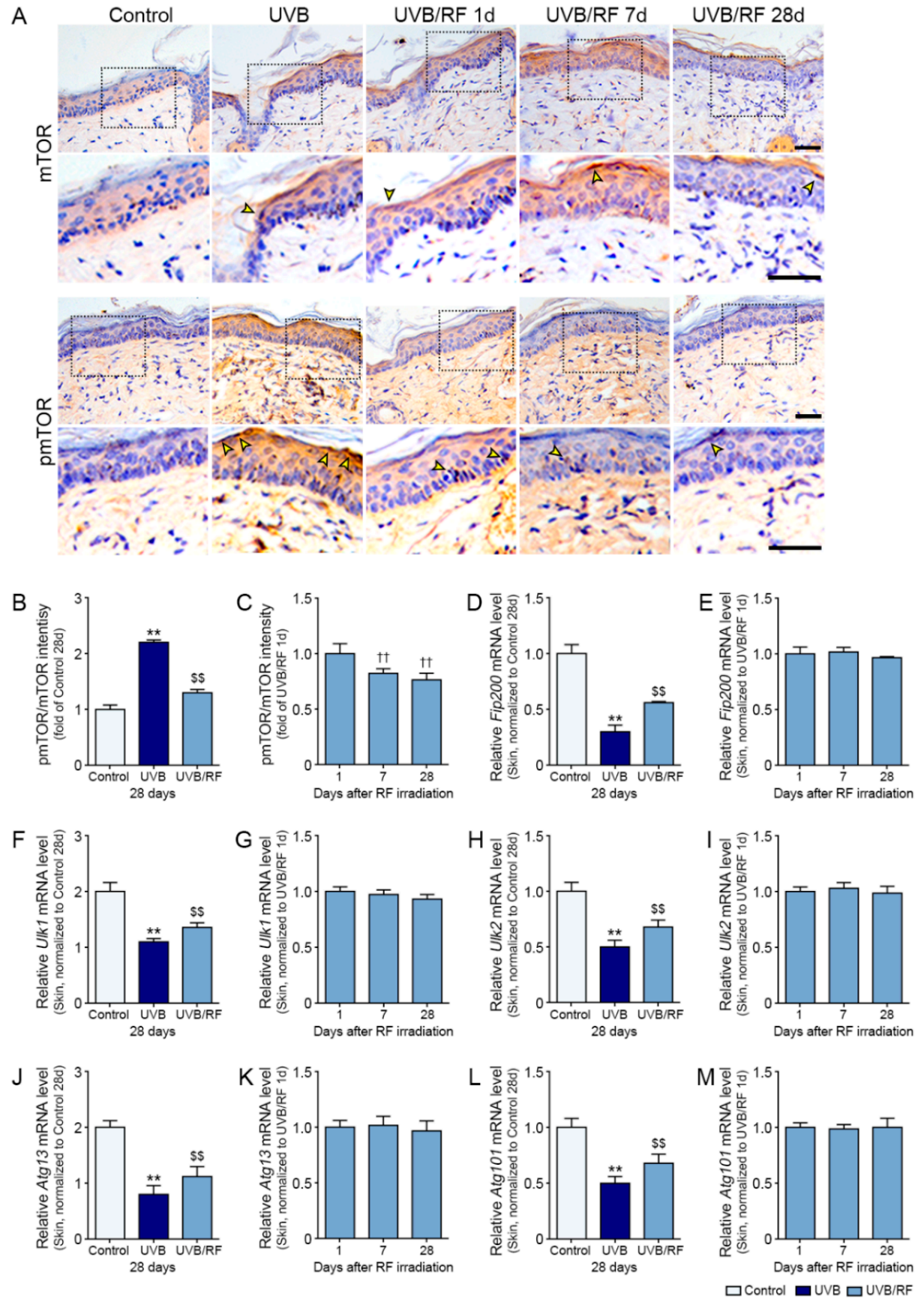

Figure 2. Activation effects of autophagy initiation response by RF irradiation in the UV-B-exposed animal model. (A) mTOR and pmTOR expression (arrows) in the epidermis of the UV-B-exposed mouse skins were assessed immunohistochemically (scale bar $=100 \mu \mathrm{m}) ;(\mathbf{B}, \mathbf{C})$ Quantitative ratios of representative mTOR and pmTOR images. Mouse skins were either with UV-B or with UV-B/RF in vivo, or otherwise not irradiated (control 28 days); (D-M) The mRNA expression levels of (D,E) FIP200, (F,G) ULK1, (H,I) ULK2, (J,K) ATG13, and (L,M) ATG101 were determined in autophagy initiation response-related factors in skin tissue. All mRNA levels were measured by qRT-PCR, normalized versus ACTB, and expressed relative to levels in the control 28 days group or 1 day after $\mathrm{RF}$ irradiation. Data are presented as mean $\pm \mathrm{SD}$. ${ }^{* *}, p<0.01$, vs. control 28 days; $\$ \$, p<0.01$, vs. UV-B 28 days; $+\dagger, p<0.01$, vs. 1 day after RF irradiation (Mann-Whitney U test). ATG, autophagyrelated protein; $\mathrm{mTOR}$, mechanistic target of rapamycin complex; pmTOR, phosphorylated mTOR; $\mathrm{RF}$, radiofrequency; ULK, unc-51-like autophagy activating kinase 1; UV-B, ultraviolet-B; UV-B/RF, UV-B plus RF. 

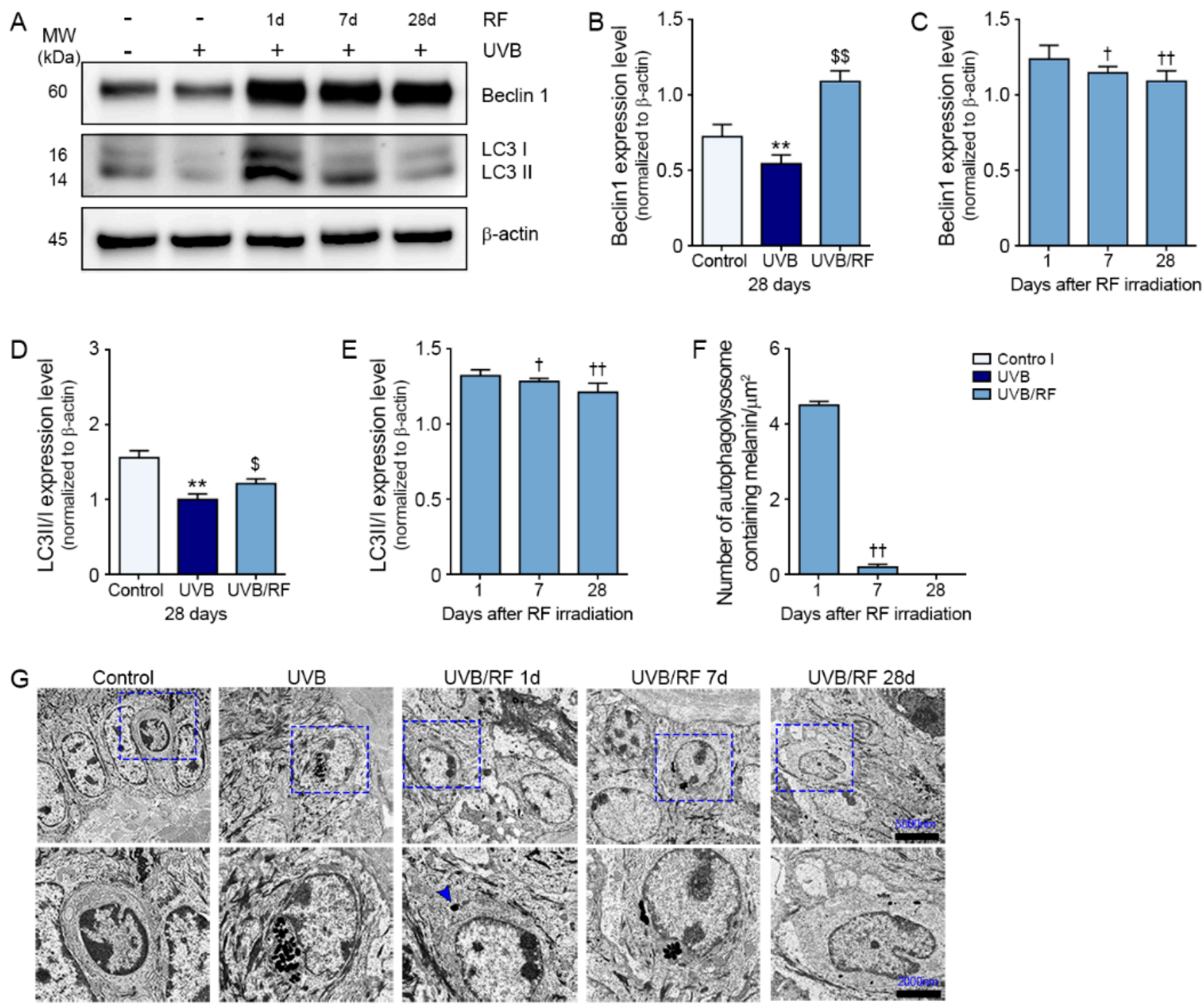

Figure 3. Activation of autophagosomes by RF irradiation in the UV-B-exposed animal model. (A) The expression levels of autophagy-related factors in skin tissue were assessed by Western blot; (B-E) Quantitative graphs of representative Western blot images. The protein expression levels were normalized to $\beta$-actin; (F) Quantitative graphs of representative TEM images; (G) Changes in autophagosome (arrows) numbers with time after RF irradiation were observed under TEM. Data are presented as mean $\pm \mathrm{SD}$. ${ }^{* *}, p<0.01$, vs. control (HEKn) or control 28 days (Skin); $\$, p<0.05 ; \$ \$, p<0.01$, vs. UV-B (HEKn) or UV-B 28 days (Skin);,$+ p<0.05 ;+\dagger, p<0.01$, vs. 1 day after RF irradiation (Mann-Whitney U test). LC3, microtubule-associated protein 1A/1B-light chain 3; RF, radiofrequency; TEM, transmission electron microscopy; UV-B, ultraviolet-B; UV-B/RF, UV-B plus RF. 


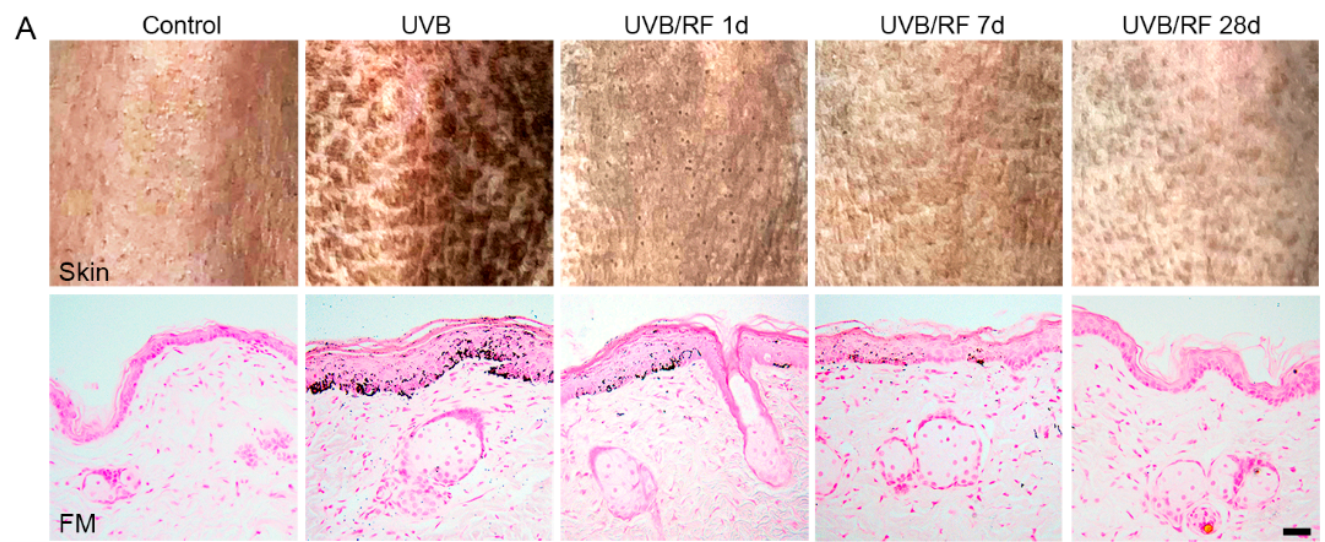

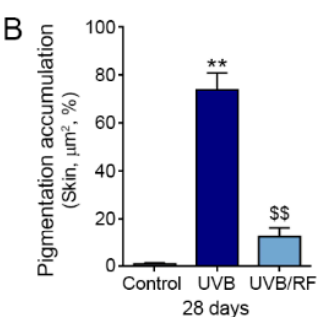

F
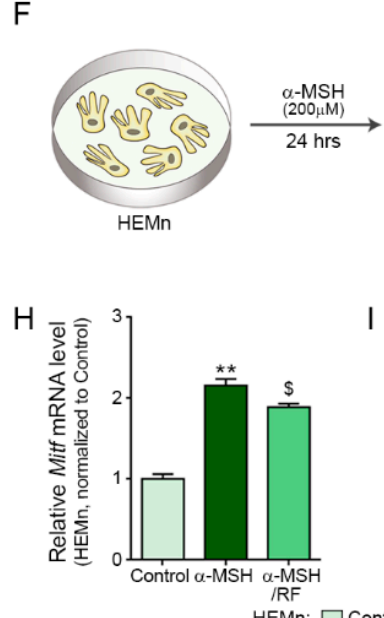

HEMn: $\square$ Contro

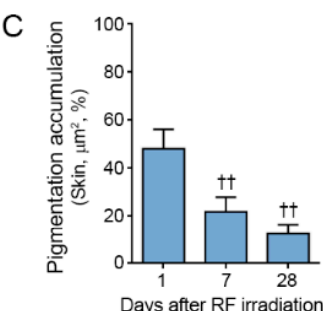

Days after RF irradiation

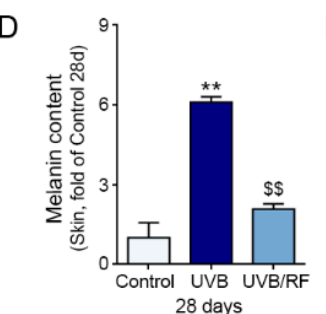

28 days
E

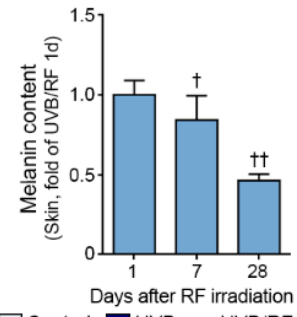

Skin:

G

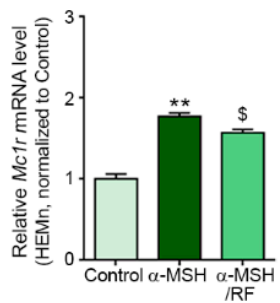

Melanin conent assay TEM

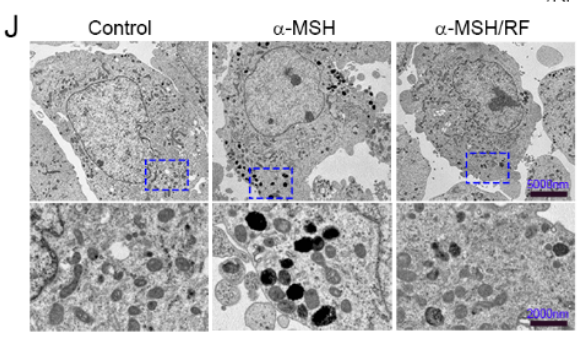

Figure 4. Reduction in melanin accumulation via autophagy activation by RF irradiation. (A) Pigmentation changes in UV-B-exposed mouse skin were assessed 1, 7, and 28 days after RF irradiation (upper row). Melanin content was assessed by FM staining (lower row; scale bar = $100 \mu \mathrm{m}$ ); (B,C) Quantitative graphs of representative mouse skin images and $(\mathbf{D}, \mathbf{E})$ representative FM staining images; (F) HEMn were treated either with $\alpha$-MSH $(200 \mu \mathrm{M})$ or with $\alpha-\mathrm{MSH} / \mathrm{RF}(2 \mathrm{MHz}, 10 \mathrm{~W}$ for $100 \mathrm{~ms})$ in vitro, or otherwise not irradiated (control); $(\mathbf{G}, \mathbf{H})$ The mRNA expression levels of MC1R (F), and MITF (G) were identified in the skin tissue. All mRNA levels were measured by qRT-PCR, normalized versus ACTB, and expressed relative to levels in the control; (I) Melanin content was measured in HEMn after RF irradiation; (J) Changes in melanin after RF irradiation were observed with TEM. Data are presented as mean \pm SD. ${ }^{* *}, p<0.01$, vs. control 28 days (skin) or control (HEMn); $\$, p<0.05 ; \$ \$, p<0.01$, vs. UV-B 28 days (Skin) or $\alpha$-MSH (HEMn);,$+ p<0.05 ;+\dagger, p<0.01$, vs. 1 day after RF irradiation (Mann-Whitney U test). $\alpha$-MSH, alpha-melanotropin; FM, Fontana-Masson staining; HEMn, human epidermal melanocyte; NF- $\kappa \mathrm{B}$, nuclear factor kappa-light-chain-enhancer of activated B cells; MC1R, melanocortin 1 receptor; MITF, micropthalmia-associated transcription factor; RF, radiofrequency; TEM, transmission electron microscopy; TNFR, tumor necrosis factor receptor; TLR4, Toll-like receptor 4; UV-B, ultraviolet-B; UV-B/RF, UV-B plus RF. 


\section{Discussion}

Even though melanin protects the skin against UV radiation [23], excessive accumulation of melanin leads to hyperpigmentation-related disorders like melasma or freckles, which cause cosmetic problems [24]. Autophagy helps determine skin color by modulating melanosome degradation in keratinocytes [8]. Autophagic activity is related to the degree of skin pigmentation wherein keratinocytes in lighter skin are more able to degrading melanosomes than keratinocytes in darker skin [25]. A previous study showed that LC3B expression and autophagy were more decreased in melanocytes of melasma lesions than in unaffected areas of the skin [26]. NF- $\mathrm{KB}$ is activated by UV radiation [27]. The expressions of TNF- $\alpha$ and TLR4 are also increased by UV radiation [17-20], both of which downregulate autophagy by activating NF-kB $[14,19,20]$. In our study, we evaluated whether RF irradiation reduced skin pigmentation by restoring autophagy, which was decreased by UV-B radiation. We found that RF irradiation downregulated the expressions of TNFR, TLR4, and NF- $\mathrm{kB}$, thereby increasing autophagic activity, an effect that was observed up to 28 days after $R F$ irradiation.

Autophagy is initiated by the generation of double membrane-bound autophagosomes. In the autophagic process, autophagosomes form autolysosomes by merging with lysosomes [28]. Various stresses activate AMP-activated protein kinase (AMPK) and inhibit the mTOR, which consequently initiates autophagy by upregulating the FIP200, ULK 1, ATG13, and ATG101 [29]. After the initiation of autophagy, phagophore nucleation follows, which involves various proteins like ATG6 (Beclin 1), ATG14, and vacuolar protein sorting-associated protein 15 (Vps15) [29]. The next stage of nucleation is phagophore elongation. During elongation, precursors of LC3-like proteins are cleaved to produce LC3-II. By conjugation with phosphatidylethanolamine, the cytosolic form of LC-I becomes LC-II, which is an autophagosome-bound form [29]. LC3-II enhances targeted degradation of aggregated proteins or injured cellular organelles by interacting with adaptor proteins, such as p62 [10]. Thus, LC3-II is frequently used to measure autophagic flux [30].

Various cellular stress signals lead to the activation of mTORC1, which is an upstream target of the autophagy core machinery and the inactivation of which initiates autophagy [31]. mTORC1 inhibition activates ULK1/2 kinase activity, and then ULK1 and ULK2 phosphorylate ATG13 and FIP200, which are essential subunits of the ULK1/2 kinase complex [31-33]. In our study, pmTOR expression was increased by UV-B radiation and conversely decreased by RF radiation. Our findings suggest that RF irradiation promotes autophagy by inactivating mTOR, which otherwise inhibits autophagy and autophagic flux. We also evaluated whether RF irradiation contributed to melanosome removal by autophagy on TEM and found that an increase in the melanin-containing autophagosomes was observed from 1 day to 7 days after RF irradiation. Melanin deposition in the skin was increased by UV-B but decreased by RF radiation. Increased skin melanin accumulation is resulted from decreased melanin removal and increased melanin synthesis. Thus, we also evaluated whether changes of melanin synthesis by RF might also involve decreasing melanogenesis, and which possible mechanism might decrease inflammatory signal pathways such as TNF by RF. For evaluating exact mechanisms for decreasing melanogenesis by RF, further study is needed.

Various treatments have been suggested to reduce melanin accumulation, such as the use of hypopigmentation agents, which include tyrosinase inhibitors such as hydroquinone and arbutin, which block melanogenesis [34]. However, such agents are not very effective and cause irritation [34]. Natural products such as marliolide or ursolic acid have been found effective in reducing melanin deposition by increasing autophagy [34,35]. These studies showed that increasing autophagic activity led to melanin degradation and thus decreased skin pigmentation.

Our study showed that RF irradiation decreased skin pigmentation by increasing autophagy in skin in the UV-B-irradiated mouse model. RF irradiation decreased the expressions of TLR4 and TNFR, which in turn decreased mTOR activity, thereby increasing autophagic activity. Since we did not use knock-out animal models, it is hard to show 
which signal pathways were definitive ones to modulate autophagy by RF. To evaluate the exact mechanism of decreasing skin pigmentation by RF, future studies with knock-out animal models are needed. Moreover, this is a preclinical study which is too early to apply directly to humans.

Nevertheless, our results showed RF leads to increased autophagy which is associated with reduced melanin accumulation in the animal model. Our findings suggest that $\mathrm{RF}$ irradiation is a promising method of decreasing skin pigmentation by modulating autophagy.

\section{Materials and Methods}

\subsection{In Vitro Model and RF Irradiation}

Human primary epidermal keratinocytes (HEKn; American Type Culture Collection, ATCC, Manassas, VA, USA) were maintained with a keratinocyte growth kit (ATCC, Manassas, VA, USA). For establishing the in vitro model in HEKn, the cells were exposed to UV-B $\left(200 \mathrm{~mJ} / \mathrm{cm}^{2}\right)$ for $5 \mathrm{~min}$, irradiated with RF (POTENZA, Jeisys Medical Inc., Seoul, Korea; $2 \mathrm{MHz}, 10 \mathrm{~W}, 100 \mathrm{~ms}$ ), and incubated for $24 \mathrm{~h}$ (Figure 1A).

Human primary epidermal melanocytes (HEMn; ATCC, Manassas, VA, USA) were grown in Dermal Cell Basal Medium (ATCC, Manassas, VA, USA) with a melanocyte growth kit (ATCC, Manassas, VA, USA). For establishing the in vitro model in HEMn, the cells were treated with $200 \mathrm{nM} \alpha$-MSH (Sigma Aldrich, St. Louis, MO, USA) and kept in an incubator at $37^{\circ} \mathrm{C}$ in an atmosphere of $5 \% \mathrm{CO}_{2}$ for $24 \mathrm{~h}$. Then, the cells were irradiated with $\mathrm{RF}$, and incubated for $48 \mathrm{~h}$ (Figure $4 \mathrm{~F}$ ).

\subsection{Measurement of Melanin Content in Cells}

To assess melanin content in HEMn, the cells were seeded at $1 \times 10^{4}$ cells/well in 96well plates and incubated for $24 \mathrm{~h}$. After applying $\alpha$-MSH and RF, the cells were harvested by centrifugation at $12,000 \times g$ for $20 \mathrm{~min}$ and dissolved in $100 \mu \mathrm{L}$ of $10 \%$ dimethyl sulfoxide (DMSO) and $1 \mathrm{~N} \mathrm{NaOH}$ solution for $20 \mathrm{~min}$ at $95^{\circ} \mathrm{C}$. Absorbance at $490 \mathrm{~nm}$ was measured with a microplate reader (Molecular Devices).

\subsection{In Vivo Model and RF Irradiation}

Five-week-old male HRM-2 mice (20-25 g) were obtained from Central Lab Animal Inc. (Seoul, Korea) and cared to adapt for 2 weeks. The mice were housed in cages under a controlled temperature $\left(23^{\circ} \mathrm{C}\right)$ with a $12 \mathrm{~h}$ light/dark cycle and free access to food and water.

After the adaptation period, the mice were randomly divided into five groups as follows: (1) control (no exposure to UV-B and no irradiated RF), (2) UV-B (exposure to UV-B at $200 \mathrm{~mJ} / \mathrm{cm}^{2}$ ), (3) UV-B/RF $1 \mathrm{~d}$ (exposure to UV-B/irradiated RF; sampling proceeds 1 day after RF irradiation), (4) UV-B/RF 7d (exposure to UV-B/irradiated RF; sampling proceeds 7 days after RF irradiation), (5) UV-B/RF 28d (exposure to UV-B/irradiated RF; sampling proceeds 28 days after RF irradiation). The mice were exposed to UV-B for $5 \mathrm{~min}$ once every 2 days for 10 days and then for 5 min every day for the next 3 days (total of 13 days) [36]. Subsequently, the mice were irradiated to $\mathrm{RF}(2 \mathrm{MHz}, 10 \mathrm{~W}$ for $100 \mathrm{~ms}$ ) and then exposed to UV-B every 2 days for 28 days. The skin tissues of mice were harvested after 1 day, 7 days and 28 days of RF irradiation (Figure 1E).

This study was approved by the Center of Animal Care and Use ethical board of Gachon University (Approval Number LCDI-2020-0115) and executed in accordance with the Institutional Animal Care and Use Committee.

\subsection{RF Irradiation System}

The irradiation system (POTENZA, Jeisys Medical Inc., Seoul, Korea) used for this study was a bipolar pulse-type electrode array radiofrequency device. An impedance matching system was used to determine the compensation value by automatically measuring impedance, and RF was applied using a 16 ea $(4 \times 4)$ needle tip. RF was administered at 
$2 \mathrm{MHz}$ using pulse-type, bipolar, alternating current oscillations in the animal experiment. Single pulse-type bipolar RF devices were used in the animal experiment and comprised an on-time pulse duration of $100 \mathrm{~ms}$ at a power density of $10 \mathrm{~W} /$ pulse. The invasive microneedle for RF application had a length of $13.6 \mathrm{~mm}$, a diameter of $250 \mathrm{~mm}$, and a needle-to-needle distance of $1.3 \mathrm{~mm}$, and irradiation and treatment were performed with a disposable tip of $10 \mathrm{~mm} \times 10 \mathrm{~mm}$ consisting of 16 electrodes. The tip was approved by NAMSA (Northwood, OH, USA) after biological compatibility testing.

\subsection{Sample Preparation \\ 4.5.1. Extraction of RNA and cDNA Synthesis}

The cells and frozen skin tissues were ground using liquid nitrogen and homogenized by the RNAiso Plus reagent (Takara, Shiga, Japan) according to the manufacturer's instructions.

The extracted RNA was quantified by the NanoDrop 2000 spectrophotometer (Thermo Fisher Scientific, Waltham, MA, USA) and was converted to cDNA using a PrimeScript $1^{\text {ST }}$ strand cDNA Synthesis Kit (Takara, Shiga, Japan) for quantitative real-time polymerase chain reaction (qRT-PCR).

\subsubsection{Paraffin-Embedded Tissue Sectioning}

The skin tissues that were fixed by $4 \%$ paraformaldehyde (Sigma-Aldrich, St. Louis, MO, USA) were washed for 30 min for embedding. Skin paraffin blocks made using a tissue processor (Thermo Fisher Scientific, Waltham, MA, USA) were sectioned at 7- $\mu \mathrm{m}$ using a microtome (Leica, Wetzlar, Germany), and cooked at $37^{\circ} \mathrm{C}$ overnight to keep them attached to the slides. The sectioned slides were passed through xylene and four concentrations of ethanol $(100 \%, 95 \%, 80 \%$, and $70 \%)$ to deparaffinate them for staining.

\subsubsection{Isolation of Protein}

The frozen skin tissues were ground using liquid nitrogen and homogenized by the RIPA buffer (EzRIPA, ATTO, Tokyo, Japan) with proteinase and phosphatase inhibitors. The homogenized skin tissues were sonicated and then centrifuged at $14,000 \times g$ for $15 \mathrm{~min}$ at $4{ }^{\circ} \mathrm{C}$ After centrifugation, the isolated protein (supernatant liquids) was aliquoted and quantified by a bicinchoninic acid assay kit (Thermo Fisher Scientific, Inc., Waltham, MA, USA).

\subsection{Quantitative Real-Time Polymerase Chain Reaction}

The qRT-PCR mixed a reagent containing the SYBR Green reagent (Takara), $1 \mu \mathrm{g}$ of synthesized cDNA template, and a 10 pmol primer (Table S1), which were dispensed into 384-well multi-plates, and then analyzed by the CFX386 Touch Real-Time PCR System (Bio-Rad, Hercules, CA, USA).

\subsection{3,3-Diaminobenzidine Staining for Immunohistochemistry Use}

The sectioned skin tissue slides were incubated in 3\% hydrogen peroxide in methanol for $30 \mathrm{~min}$ at room temperature to block endogenous peroxidase. The tissue slides were washed using a phosphate-buffered saline (PBS) and then incubated with mTOR antibodies (1:400; LSBio, Seattle, WA, USA) and pmTOR antibodies (1:50; Santa Cruz Biotechnology Inc., Dallas, TX, USA) in normal serum for $24 \mathrm{~h}$ at $4{ }^{\circ} \mathrm{C}$. The slides were rinsed with PBS and incubated with a biotinylated secondary antibody using the $\mathrm{ABC}$ kit (Vector Laboratories Inc., Burlingame, CA, USA) for $2 \mathrm{~h}$ at room temperature. After washing with PBS, the tissue slides were developed using 3,3'-diaminobenzidine (Sigma-Aldrich) for $15 \mathrm{~min}$ to confirm the brown signal. To identify nuclei, tissue slides were stained in hematoxylin solution for $1 \mathrm{~min}$, then mounted with dibutylphthalate polystyrene xylene mounting solution (Sigma-Aldrich). Images of the stained tissues were taken under an optical microscope (Olympus Optical Co., Tokyo, Japan) and analyzed using ImageJ software (NIH, Bethesda, MD, USA). 


\subsection{Western Blotting}

Equal amounts of isolated skin proteins were separated on 8-12\% polyacrylamide gels and transferred to polyvinylidene fluoride membranes (Millipore, Burlington, MA, USA) by a power station (ATTO, Osaka, Japan). After blocking using $5 \%$ skim milk and washing with Tris-buffered saline with $0.1 \%$ Tween 20 (TTBS), the membranes were incubated with Beclin 1 antibodies (1:2000; Bioss, Woburn, MA, USA), LC3 (1:500; Bioss) and $\beta$-actin (1:1000; Cell Signaling, Danvers, MA, USA) for $12 \mathrm{~h}$ at $4{ }^{\circ} \mathrm{C}$ and then washed with TTBS. The membranes were then incubated with a secondary antibody (Vector Laboratories, Burlingame, CA, USA) and rinsed with TTBS. Subsequently, an enhanced chemiluminescence detection reagent (GE Healthcare, Chicago, IL, USA) was used to visualize the immunoreactive proteins on the membrane.

\subsection{Transmission Electron Microscopy}

Specimens were fixed for $12 \mathrm{~h}$ in $2 \%$ glutaraldehyde $/ 2 \%$ paraformaldehyde in $0.1 \mathrm{M}$ phosphate buffer ( $\mathrm{pH} 7.4$ ) and washed in $0.1 \mathrm{M}$ phosphate buffer, post-fixed with $1 \% \mathrm{OsO}_{4}$ in $0.1 \mathrm{M}$ phosphate buffer for $2 \mathrm{~h}$, dehydrated with an ascending ethanol series $(50 \%, 60 \%$, $70 \%, 80 \%, 90 \%, 95 \%, 100 \%$, and $100 \%$ ) for $10 \mathrm{~min}$ each, and infiltrated with propylene oxide for $10 \mathrm{~min}$.

The fixed samples were embedded using a Poly/Bed 812 kit (Polysciences, Warrington, PA, USA) and polymerized in an electron microscope oven (DOSAKA, Katsumi, Japan) at $65^{\circ} \mathrm{C}$ for $12 \mathrm{~h}$. The block was equipped with a diamond knife in the ultramicrotome, cut into $200 \mathrm{~nm}$ sections, and stained with toluidine blue for optical microscopy.

The region of interest was then cut into $80 \mathrm{~nm}$ sections using the ultramicrotome, placed on copper grids, double stained with $3 \%$ uranyl acetate for $30 \mathrm{~min}$ and $3 \%$ lead citrate for $7 \mathrm{~min}$, and observed under a TEM (JEOL, Tokyo, Japan) equipped with a Megaview III CCD camera (Soft Imaging System-Germany) at an acceleration voltage of $80 \mathrm{kV}$.

\subsection{Fontana-Masson Staining}

The skin tissues were incubated in Fontana ammoniacal silver solution (ScyTek, West Logan, UT, USA) overnight at room temperature, subsequently rinsed three times with distilled water, and then incubated in hypo solution for $3 \mathrm{~min}$. Afterwards, the tissues were washed in distilled water, counterstained with neutral red stain for $5 \mathrm{~min}$, washed in distilled water, dehydrated in absolute alcohol, and mounted for observation.

\subsection{Statistical Analysis}

We performed a Kruskal-Wallis test for comparisons of three groups, followed by a Mann-Whitney U test as a post hoc test. This study was validated using an unpaired $t$-test. All results are presented as mean \pm standard deviation, and the statistical significance was displayed: *, vs. control (HEKn or HEMn) or control 28 days (skin); \$, vs. UV-B (HEKn) or $\alpha$-MSH (HEMn) or UV-B 28 days (skin); †, vs. UV-B/RF 1 day (skin). All statistical analyses were performed using SPSS version 22 (IBM Corporation; Armonk, NY, USA).

Supplementary Materials: The following are available online at https:/ / www.mdpi.com/article / 10.3390/ijms221910724/s1, Table S1: Primer list for quantitative polymerase chain reaction used in this study.

Author Contributions: Conceptualization, H.M.K., K.H.S. and K.B.; validation, S.O. and J.Y.Y.; formal analysis, H.M.K., S.O., J.Y.Y., H.J.S. and M.J.; investigation, H.M.K., S.O. and K.H.S.; resources, H.J.S., M.J., D.K. and K.B.; data curation, H.M.K., S.O., J.Y.Y., H.J.S., M.J. and D.K.; writing—original draft preparation, H.M.K., S.O. and K.H.S.; writing-review and editing, H.M.K., S.O., D.K., K.H.S. and K.B.; visualization, S.O. and K.B.; supervision, K.H.S. and K.B.; project administration, K.H.S. and K.B.; funding acquisition, K.B. All authors have read and agreed to the published version of the manuscript.

Funding: This research was funded by Jeisys Medical Inc. (Grant no. 2020-5004). 
Institutional Review Board Statement: The study was conducted according to the guidelines of the Institutional Animal Care and Use Committee, and approved by the Institutional Review Board (or Ethics Committee) of Gachon University (approval No. LCDI-2020-0115).

Informed Consent Statement: Not applicable.

Data Availability Statement: All data is contained within the article.

Acknowledgments: Jeisys Medical Inc., (Seoul, Korea) provided us with the POTENZA microneedlingradiofrequency machine.

Conflicts of Interest: The authors have no conflict of interest to declare.

\section{References}

1. D'Mello, S.A.; Finlay, G.J.; Baguley, B.C.; Askarian-Amiri, M.E. Signaling Pathways in Melanogenesis. Int. J. Mol. Sci. 2016, 17, 1144. [CrossRef]

2. Hseu, Y.C.; Vudhya Gowrisankar, Y.; Wang, L.W.; Zhang, Y.Z.; Chen, X.Z.; Huang, P.J.; Yen, H.R.; Yang, H.L. The in vitro and in vivo depigmenting activity of pterostilbene through induction of autophagy in melanocytes and inhibition of UVA-irradiated $\alpha$-MSH in keratinocytes via Nrf2-mediated antioxidant pathways. Redox Biol. 2021, 44, 102007. [CrossRef] [PubMed]

3. Quevedo, W.C., Jr.; Holstein, T.J. Molecular genetics and the ontogeny of pigment patterns in mammals. Pigment Cell Res. 1992, 5, 328-334. [CrossRef] [PubMed]

4. Ebanks, J.P.; Wickett, R.R.; Boissy, R.E. Mechanisms regulating skin pigmentation: The rise and fall of complexion coloration. Int. J. Mol. Sci. 2009, 10, 4066-4087. [CrossRef]

5. Chen, L.; Xu, Z.; Jiang, M.; Zhang, C.; Wang, X.; Xiang, L. Light-emitting diode 585 nm photomodulation inhibiting melanin synthesis and inducing autophagy in human melanocytes. J. Dermatol. Sci. 2018, 89, 11-18. [CrossRef] [PubMed]

6. Lin, J.Y.; Fisher, D.E. Melanocyte biology and skin pigmentation. Nature 2007, 445, 843-850. [CrossRef] [PubMed]

7. Zhou, Y.Y.; Li, Y.; Jiang, W.Q.; Zhou, L.F. MAPK/JNK signalling: A potential autophagy regulation pathway. Biosci. Rep. 2015, 35, e00199. [CrossRef] [PubMed]

8. Murase, D.; Hachiya, A.; Takano, K.; Hicks, R.; Visscher, M.O.; Kitahara, T.; Hase, T.; Takema, Y.; Yoshimori, T. Autophagy has a significant role in determining skin color by regulating melanosome degradation in keratinocytes. J. Invest. Dermatol. 2013, 133, 2416-2424. [CrossRef]

9. Katsuyama, Y.; Taira, N.; Yoshioka, M.; Okano, Y.; Masaki, H. Disruption of melanosome transport in melanocytes treated with theophylline causes their degradation by autophagy. Biochem. Biophys. Res. Commun. 2017, 485, 126-130. [CrossRef]

10. Kim, Y.C.; Guan, K.L. mTOR: A pharmacologic target for autophagy regulation. J. Clin. Invest. 2015, 125, 25-32. [CrossRef] [PubMed]

11. Djavaheri-Mergny, M.; Amelotti, M.; Mathieu, J.; Besançon, F.; Bauvy, C.; Codogno, P. Regulation of autophagy by NFkappaB transcription factor and reactives oxygen species. Autophagy 2007, 3, 390-392. [CrossRef]

12. Xiao, G. Autophagy and NF-kappaB: Fight for fate. Cytokine Growth Factor Rev. 2007, 18, 233-243. [CrossRef] [PubMed]

13. Morselli, E.; Galluzzi, L.; Keep, O.; Vicencio, J.M.; Criollo, A.; Maiuri, M.C.; Kroemer, G. Anti- and pro-tumor functions of autophagy. Biochim. Biophys. Acta 2009, 1793, 1524-1532. [CrossRef] [PubMed]

14. Chaturvedi, M.M.; Sung, B.; Yadav, V.R.; Kannappan, R.; Aggarwal, B.B. NF-kB addiction and its role in cancer: One size does not fit all. Oncogene 2011, 30, 1615-1630. [CrossRef]

15. Xiao, G.; Fu, J. NF-kB and cancer: A paradigm of Yin-Yang. Am. J. Cancer Res. 2011, 1, 192-221.

16. Djavaheri-Mergny, M.; Amelotti, M.; Mathieu, J.; Besançon, F.; Bauvy, C.; Souquère, S.; Pierron, G.; Codogno, P. NF-kappaB activation represses tumor necrosis factor-alpha-induced autophagy. J. Biol. Chem. 2006, 281, 30373-30382. [CrossRef]

17. Zhou, M.; Xu, W.; Wang, J.; Yan, J.; Shi, Y.; Zhang, C.; Ge, W.; Wu, J.; Du, P.; Chen, Y. Boosting mTOR-dependent autophagy via upstream TLR4-MyD88-MAPK signalling and downstream NF- $\mathrm{kB}$ pathway quenches intestinal inflammation and oxidative stress injury. EBioMedicine 2018, 35, 345-360. [CrossRef] [PubMed]

18. Freedberg, I.M.; Tomic-Canic, M.; Komine, M.; Blumenberg, M. Keratins and the keratinocyte activation cycle. J. Invest. Dermatol. 2001, 116, 633-640. [CrossRef]

19. Wu, L.; Chen, X.; Zhao, J.; Martin, B.; Zepp, J.A.; Ko, J.S.; Gu, C.; Cai, G.; Ouyang, W.; Sen, G.; et al. A novel IL-17 signaling pathway controlling keratinocyte proliferation and tumorigenesis via the TRAF4-ERK5 axis. J. Exp. Med. 2015, 212, 1571-1587. [CrossRef]

20. Nygaard, U.; van den Bogaard, E.H.; Niehues, H.; Hvid, M.; Deleuran, M.; Johansen, C.; Vestergaard, C. The “Alarmins" HMBG1 and IL-33 Downregulate Structural Skin Barrier Proteins and Impair Epidermal Growth. Acta Derm. Venereol. 2017, 97, 305-312. [CrossRef]

21. Kim, H.M.; Oh, S.; Yoon, J.H.; Kang, D.; Son, M.; Byun, K. Radiofrequency Irradiation Attenuates High-Mobility Group Box 1 and Toll-like Receptor Activation in Ultraviolet B-Induced Skin Inflammation. Molecules 2021, 26, 1297. [CrossRef]

22. Alexis, A.F.; Sergay, A.B.; Taylor, S.C. Common dermatologic disorders in skin of color: A comparative practice survey. Cutis 2007, 80, 387-394. 
23. Brenner, M.; Hearing, V.J. The protective role of melanin against UV damage in human skin. Photochem. Photobiol. 2008, 84, 539-549. [CrossRef]

24. Kurita, M.; Kato, H.; Yoshimura, K. A therapeutic strategy based on histological assessment of hyperpigmented skin lesions in Asians. J. Plast. Reconstr. Aesthet. Surg. 2009, 62, 955-963. [CrossRef] [PubMed]

25. Murase, D.; Kusaka-Kikushima, A.; Hachiya, A.; Fullenkamp, R.; Stepp, A.; Imai, A.; Ueno, M.; Kawabata, K.; Takahashi, Y.; Hase, T.; et al. Autophagy Declines with Premature Skin Aging resulting in Dynamic Alterations in Skin Pigmentation and Epidermal Differentiation. Int. J. Mol. Sci. 2020, 21, 5708. [CrossRef] [PubMed]

26. Espósito, A.C.C.; de Souza, N.P.; Miot, L.D.B.; Miot, H.A. Deficit in autophagy: A possible mechanism involved in melanocyte hyperfunction in melasma. Indian J. Dermatol. Venereol. Leprol. 2021, 87, 585-586. [CrossRef] [PubMed]

27. Devary, Y.; Rosette, C.; DiDonato, J.A.; Karin, M. NF-kappa B activation by ultraviolet light not dependent on a nuclear signal. Science 1993, 261, 1442-1445. [CrossRef]

28. Ho, H.; Ganesan, A.K. The pleiotropic roles of autophagy regulators in melanogenesis. Pigment Cell Melanoma Res. 2011, 24, 595-604. [CrossRef]

29. Ravikumar, B.; Sarkar, S.; Davies, J.E.; Futter, M.; Garcia-Arencibia, M.; Green-Thompson, Z.W.; Jimenez-Sanchez, M.; Korolchuk, V.I.; Lichtenberg, M.; Luo, S.; et al. Regulation of mammalian autophagy in physiology and pathophysiology. Physiol. Rev. 2010, 90, 1383-1435. [CrossRef]

30. Pankiv, S.; Clausen, T.H.; Lamark, T.; Brech, A.; Bruun, J.A.; Outzen, H.; Øvervatn, A.; Bjørkøy, G.; Johansen, T. p62/SQSTM1 binds directly to Atg8/LC3 to facilitate degradation of ubiquitinated protein aggregates by autophagy. J. Biol. Chem. 2007, 282, 24131-24145. [CrossRef]

31. Hosokawa, N.; Hara, T.; Kaizuka, T.; Kishi, C.; Takamura, A.; Miura, Y.; Iemura, S.; Natsume, T.; Takehana, K.; Yamada, N.; et al. Nutrient-dependent mTORC1 association with the ULK1-Atg13-FIP200 complex required for autophagy. Mol. Biol. Cell 2009, 20, 1981-1991. [CrossRef] [PubMed]

32. Ganley, I.G.; Lam, d.H.; Wang, J.; Ding, X.; Chen, S.; Jiang, X. ULK1.ATG13.FIP200 complex mediates mTOR signaling and is essential for autophagy. J. Biol. Chem. 2009, 284, 12297-12305. [CrossRef]

33. Jung, C.H.; Jun, C.B.; Ro, S.H.; Kim, Y.M.; Otto, N.M.; Cao, J.; Kundu, M.; Kim, D.H. ULK-Atg13-FIP200 complexes mediate mTOR signaling to the autophagy machinery. Mol. Biol. Cell 2009, 20, 1992-2003. [CrossRef] [PubMed]

34. Yun, C.Y.; Choi, N.; Lee, J.U.; Lee, E.J.; Kim, J.Y.; Choi, W.J.; Oh, S.H.; Sung, J.H. Marliolide Derivative Induces Melanosome Degradation via Nrf2/p62-Mediated Autophagy. Int. J. Mol. Sci. 2021, 22, 3995. [CrossRef] [PubMed]

35. Park, H.J.; Jo, D.S.; Choi, D.S.; Bae, J.E.; Park, N.Y.; Kim, J.B.; Chang, J.H.; Shin, J.J.; Cho, D.H. Ursolic acid inhibits pigmentation by increasing melanosomal autophagy in B16F1 cells. Biochem. Biophys. Res. Commun. 2020, 531, 209-214. [CrossRef] [PubMed]

36. Chung, K.W.; Jeong, H.O.; Jang, E.J.; Choi, Y.J.; Kim, D.H.; Kim, S.R.; Lee, K.J.; Lee, H.J.; Chun, P.; Byun, Y.; et al. Characterization of a small molecule inhibitor of melanogenesis that inhibits tyrosinase activity and scavenges nitric oxide (NO). Biochim. Biophys. Acta 2013, 1830, 4752-4761. [CrossRef] 